\title{
Synchronization control of two identical three restricted body problems via active control
}

\author{
Mohd Arif ${ }^{1}$ and Ravi Kumar Sagar ${ }^{2}$ \\ ${ }^{1}$ Department of Mathematics, Zakir Husain Delhi College, New Delhi, India \\ ${ }^{2}$ Department of Mathematics, University of Delhi, New Delhi, India
}

Received: 26 October 2017, Accepted: 12 January 2018

Published online: 10 August 2018.

\begin{abstract}
This article presents the chaos synchronization problem of the restricted three body problem (RTBP) when the primaries are moving in a circular orbit around their center of mass in the non-uniform motion. The feedback controller for the stability of the closed-loop system is designed using the active control strategy. Simulation results satisfy the theoretical findings. For validation of results by numerical simulations, the mathematica 10 is used when the primaries are Mars and Earth.
\end{abstract}

Keywords: Space dynamics, Restricted three body problem, Complete synchronization, Lyapunov stability theory and Routh- Hurwitz criteria.

\section{Introduction}

A deterministic system is chaotic whenever its evolution sensitively depends on the initial conditions. This property implies that two trajectories emerging from near by initial conditions separate exponentially in the course of time. In 1990, Pecora and Carroll [24] gave idea of synchronization of chaotic systems using the concept of master and slave system and they demonstrated that chaotic synchronization could be achieved by driving or replacing one of the variables of a chaotic system with a variable of another similar chaotic device. Many methods and techniques for chaos control and synchronization of various chaotic systems have been developed, such as non linear feedback control [20], OGY approach [22], sliding mode control [11], adaptive synchronization [33], anti synchronization method [23], active control [4] and so on.

The active control is an efficient technique for synchronizing the chaotic systems. This method has been applied to many practical systems such as spatiotemporal dynamical systems (Codreanu [8]), the Rikitake two-disc dynamo-a geographical systems (Vincent [32] ), Non-linear Bloch equations modeling "jerk" equation and R. C. L shunted Josephson junctions (Ucar et al. [30,31] ), Complex dynamos (Mahmoud [21] ), Qi systems (Lei et al. [18]) and Hyper-chaotic and time delay systems (Israr Ahmad et al. [1,2]) etc.

Extensive research work has been devoted to address the circular restricted three body problem in the field of astronomy and space dynamics. A numbers of good research papers have investigated the circular restricted three body problem such as the Euler [10], Hill [12], Poincare [25], Birkhoff [5], Whittaker [35], Wintner [34], Lagrange [17], Deprit [9], Hadjidemetriou [13], Bhatnagar [7,8], Sharma et al. [27,28], Sahoo and Ishwar [26] and many others. These studies focus on the analytical, qualitative and numerical studies of the problem. A detailed analysis of this problem is illustrated 
in the work of American mathematician Szebehely [29].

Khan and Shahzad [14] investigated the synchronization behaviour of the two identical circular restricted three body problem influenced by radiation evolving from different initial conditions via the active control. In 2013, Khan and Tripathi [15] have investigated the synchronization behavior of a restricted three body problem under the effect of radiation pressure. In an another paper the Complete synchronization, anti-synchronization and hybrid synchronization of two identical parabolic restricted three body problem have been studied by Khan and Rimpi pal [16]. Arif [3] studied the complete synchronization, anti-synchronization and hybrid synchronization in the planar restricted three problem by taking into consideration the small primary is ellipsoid and bigger primary an oblate spheroid via active control technique.

Being motivated by the above discussion, in this article, the equation of motion of the restricted three body problem when the primaries are moving in a circular orbit around their center of mass in the orbit-orbit resonance (not in uniform motion) is formulated. This paper also discusses the complete synchronization behavior of this problem by active control techniques. It has been observed that the system is chaotic for some values of parameter. Hence the slave chaotic system completely traces the dynamics of the master system in the course of time. The paper is organized as follows. In section 2 we derive the equations of motion when the primaries moving in a circular orbit around their center of mass in the non-uniform motion. Section 3 deals with the complete synchronization of the problem via active control. Finally, we conclude the paper in section 4 .

\section{Equation of motion}

In basic terms a resonance can arise when there is a simple numerical relationship between frequencies or periods. The periods involved could be the rotational and orbital periods of a single body, as in the case of spin-orbit coupling, or perhaps the orbital periods of two or more bodies, as in the case of orbit-orbit coupling. The most obvious examples of orbit-orbit resonance in the solar system are Dione-Enceladus (Saturn's moons), Hyperion-Titan (Saturn's moons), Ganymede-Europa-Io (Jupiter's moons, ratio of orbits) and between all planets etc.

In formulating the problem assuming that the two primaries of masses $m_{1}$ and $m_{2}$ are moving in the circular motion around their centre of mass $O$ with angular velocities, the mean motions $n_{1}$ and $n_{2}$ respectively Fig(1). The motion of a particle $\mathrm{P}$ of mass $\mathrm{m}$ defined by its radius vector $\bar{r}$ will be referred to a frame of reference $O \bar{x} \bar{y}$ that rotates in the same direction and the same angular velocity $n_{1}$ as the primary of mass $m_{1}$, which in this frame are taken to stay at rest on $x$-axis.

In this article authors define the relation between gravitational and centrifugal forces as

$$
\frac{k^{2} m_{1} m_{2}}{l^{2}}=a m_{1} n_{1}^{2}=b m_{2} n_{2}^{2}
$$

$k=$ Gaussian constant, $l^{2}=a^{2}+b^{2}-2 a b \cos \left(n_{1}-n_{2}\right) t^{*}=$ variable mutual distance between the primaries.

Then, the equation of motion of the particle $\mathrm{P}$ in the sidereal system may be written as:

$$
\frac{d^{2} X}{d t^{*} 2}=\frac{\partial F\left(X, Y, t^{*}\right)}{\partial X}
$$

(C) 2018 BISKA Bilisim Technology 


$$
\frac{d^{2} Y}{d t^{*} 2}=\frac{\partial F\left(X, Y, t^{*}\right)}{\partial Y}
$$

where

$$
F=k^{2}\left[\frac{m_{1}}{R_{1}}+\frac{m_{2}}{R_{2}}\right]
$$

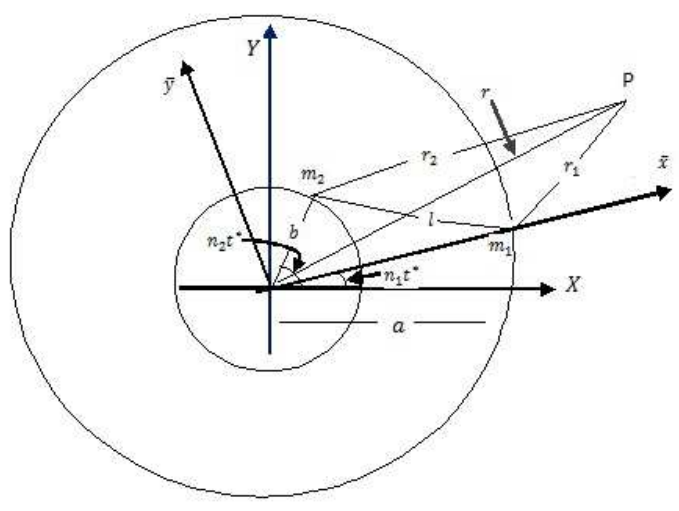

Fig. 1

$R_{1}^{2}=\left(X-X_{1}\right)^{2}+\left(Y-Y_{1}\right)^{2}, \quad R_{1}^{2}=\left(X-X_{2}\right)^{2}+\left(Y-Y_{2}\right)^{2}, \quad X_{1}=a \cos n_{1} t^{*}, \quad Y_{1}=a \sin n_{1} t^{*}, \quad X_{2}=b \cos n_{2} t^{*}, \quad Y_{2}=$ $b \sin n_{2} t^{*}$ and $t^{*}$ is the dimensional time. Now introducing a rotating co-ordinate system $(\bar{x}, \bar{y})$ by substituting

$$
Z=z e^{i n_{1} t^{*}}
$$

where $Z=X+i Y$ and $z=x+i y$. After, using the complex vector $Z$, the equation of motion (2) and (3) take the form:

$$
\frac{d^{2} Z}{d t^{*} 2}=\left(\frac{d^{2} z}{d t^{*} 2}+2 i n_{1} \frac{d z}{d t^{*}}-n_{1}^{2} z\right) e^{i n_{1} t^{*}}=-k^{2}\left[\frac{m_{1}(z-a)}{R_{1}^{3}}+\frac{m_{2}\left(z-b e^{\left(n_{2}-n_{1}\right) t^{*}}\right)}{R_{2}^{3}}\right] e^{i n_{1} t^{*}}
$$

where, $R_{1}=\left|z-z_{1}\right|, R_{2}=\left|z-z_{2}\right|, z_{1}=a e^{i n_{1} t^{*}}$ and $z_{2}=a e^{i n_{2} t^{*}}$.

Thus, the equations of motion in the rotating coordinate system are:

$$
\begin{gathered}
\frac{d^{2} \bar{x}}{d t^{*} 2}-2 n_{1} \frac{d \bar{y}}{d t^{*}}-n_{1}^{2} \bar{x}=-k^{2}\left[\frac{m_{1}(\bar{x}-a)}{\bar{r}^{3}}+\frac{m_{2}\left(\bar{x}-b \cos \left(n_{2}-n_{1}\right) t^{*}\right)}{\bar{r}^{3}}\right], \\
\frac{d^{2} \bar{y}}{d t^{*} 2}+2 n_{1} \frac{d \bar{x}}{d t^{*}}-n_{1}^{2} \bar{y}=-k^{2}\left[\frac{m_{1} \bar{y}}{\bar{r}^{3}}+\frac{m_{2}\left(\bar{y}-b \sin \left(n_{2}-n_{1}\right) t^{*}\right)}{\bar{r}^{3}}\right],
\end{gathered}
$$

$\overline{r_{1}}=\left[(\bar{x}-a)^{2}+\bar{y}^{2}\right]^{\frac{1}{2}}, \quad \overline{r_{2}}=\left[\bar{x}^{2}+\bar{y}^{2}+b^{2}-2 \bar{y} b \sin \left(n_{2}-n_{1}\right) t^{*}-2 \bar{x} b \cos \left(n_{2}-n_{1}\right) t^{*}\right]^{\frac{1}{2}}$.

The equations of motion (7) and (8) contain $k^{2}, m_{1}, m_{2}, a, b, n_{1}$, and $n_{2}$ as physical parameters which are dependent to 
each others. To reduce these parameters, now introducing dimensionless pulsating co-ordinates system given by:

$$
x=\frac{\bar{x}}{l}, \quad y=\frac{\bar{y}}{l}, \quad \mu_{1}=\frac{a}{l}, \quad \mu_{2}=\frac{b}{l}, \quad r_{1}=\frac{\overline{r_{1}}}{l}, \quad r_{2}=\frac{\overline{r_{2}}}{l} .
$$

Introducing a particular case

$$
\frac{n_{1}}{n_{2}}=\frac{1}{2}
$$

then, $\left(n_{2}-n_{1}\right) t^{*}=n_{1} t^{*}=t$. (Primaries are in a 1:2 resonance, the inner primary will complete two periods for every one period of outer primary)

Now substituting the values in equations (7) and (8), the equations of motion are

$$
\begin{aligned}
& \ddot{x}+\frac{2}{l} \dot{l} \dot{x}-2 n_{1} \dot{y}=\frac{1}{l}\left[U_{x}+2 n_{1} y \dot{l}\right], \\
& \ddot{y}+\frac{2}{l} \dot{l} \dot{y}-2 n_{1} \dot{x}=\frac{1}{l}\left[U_{y}+2 n_{1} x \dot{l}\right],
\end{aligned}
$$

where dots denote the derivatives with respect to the dimensionless time (t) and subscripts signify partial derivatives and

$$
\begin{array}{r}
U=\frac{n_{1}^{2}}{2}\left[l\left(x^{2}+y^{2}\right)+l^{2}\left(\frac{4 \mu_{2}}{r_{1}}+\frac{\mu_{1}}{r_{2}}\right)\right]-\frac{\left(x^{2}+y^{2}\right)}{2 l} \ddot{l}, \quad r_{1}^{2}=\left[\left(x-\mu_{1}\right)^{2}+y^{2}\right] \\
\text { and } \quad r_{2}^{2}=\left[x^{2}+y^{2}+\mu_{2}^{2}-2 y \mu_{2} \sin t-2 x \mu_{2} \cos t\right] .
\end{array}
$$

\section{Complete synchronization via active control}

Now to use active control techniques. Let $x=x_{1}, \quad \dot{x}=x_{2}, \quad y=x_{3}, \quad \dot{y}=x_{4}$, then, the equation (9) and (10) can be written as:

$$
\begin{gathered}
\dot{x}_{1}=x_{2}, \\
\dot{x}_{2}=x_{1}\left(n_{1}^{2}-\frac{\ddot{l}}{l}\right)-\frac{2}{l} \dot{l}_{2}+\frac{2 n_{1} x_{3} \dot{l}}{l}+2 n_{1} x_{4}+A_{1}, \\
\dot{x}_{3}=x_{4}, \\
\dot{x}_{4}=-\frac{2 n_{1} x_{1} i}{l}-2 n_{1} x_{2}+x_{3}\left(n_{1}^{2}-\frac{\ddot{l}}{l}\right)-\frac{2}{l} \dot{l}_{x_{4}}+B_{1},
\end{gathered}
$$

$A_{1}=-n_{1}^{2} l\left[\frac{4\left(x_{1}-\mu_{1}\right) \mu_{2}}{r_{1}^{3}}+\frac{\left(x_{1}-\mu_{2} \cos t\right) \mu_{1}}{r_{2}^{3}}\right], \quad B_{1}=-n_{1}^{2} l\left[\frac{4 x_{3} \mu_{2}}{r_{1}^{3}}+\frac{\left(x_{3}-\mu_{2} \sin t\right) \mu_{1}}{r_{2}^{3}}\right], \quad r_{1}^{2}=\left(x_{1}-\mu_{1}\right)^{2}+x_{3}^{2} \quad$ and $r_{2}^{2}=\left[x_{1}^{2}+x_{3}^{2}+\mu_{2}^{2}-2 x_{3} \mu_{2} \sin t-2 x_{1} \mu_{2} \cos t\right]$. 
The system (11-14) is the master system. The state orbits of this master system are shown in Figure (2a) and the surface of section in (2b) these figures shows that the system is chaotic.

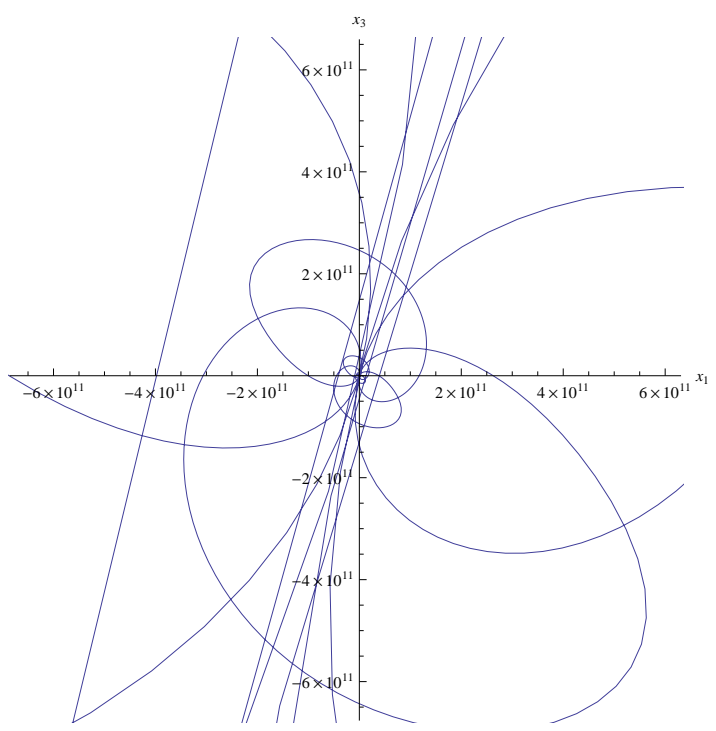

(a) State orbits

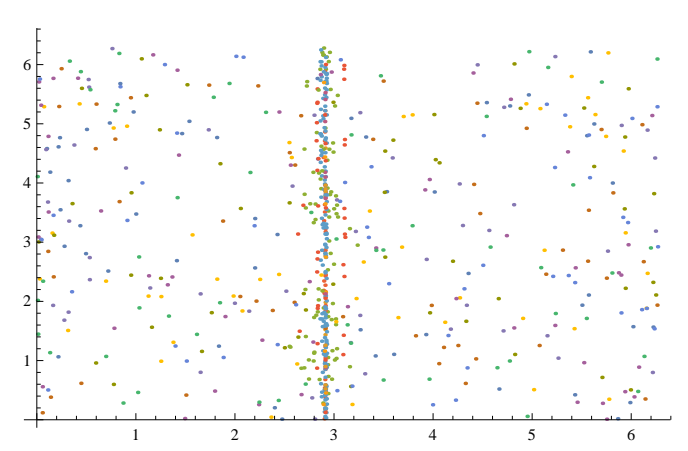

(b) Surface of section

Fig. 2

Corresponding to above master system the identical slave system is defined as:

$$
\begin{gathered}
\dot{y}_{1}=y_{2}+u_{1}(t), \\
\dot{y}_{2}=y_{1}\left(n_{1}^{2}-\frac{\ddot{l}}{l}\right)-\frac{2}{l} \dot{l}_{y_{2}}+\frac{2 n_{1} y_{3} \dot{l}}{l}+2 n_{1} y_{4}+A_{1}+u_{2}(t), \\
\dot{y}_{3}=y_{4}+u_{3}(t), \\
\dot{y}_{4}=-\frac{2 n_{1} y_{1} \dot{l}}{l}-2 n_{1} y_{2}+y_{3}\left(n_{1}^{2}-\frac{\ddot{l}}{l}\right)-\frac{2}{l} \dot{l}_{y_{4}}+B_{1}+u_{4}(t),
\end{gathered}
$$

$A_{2}=-n_{1}^{2} l\left[\frac{4\left(y_{1}-\mu_{1}\right) \mu_{2}}{r_{1}^{3}}+\frac{\left(y_{1}-\mu_{2} \cos t\right) \mu_{1}}{r_{2}^{3}}\right], \quad B_{2}=-n_{1}^{2} l\left[\frac{4 y_{3} \mu_{2}}{r_{1}^{3}}+\frac{\left(y_{3}-\mu_{2} \sin t\right) \mu_{1}}{r_{2}^{3}}\right], \quad r_{1}^{2}=\left(y_{1}-\mu_{1}\right)^{2}+y_{3}^{2}$ and $r_{2}^{2}=\left[y_{1}^{2}+y_{3}^{2}+\mu_{2}^{2}-\right.$ $\left.2 y_{3} \mu_{2} \sin t-2 y_{1} \mu_{2} \cos t\right] . u_{i}(t) ; i=1,2,3,4$ are control functions to be determined. Let $e_{i}=y_{i}-x_{i} ; i=1,2,3,4$ be the synchronization errors (Liu [19]). From (11) to (18), we obtain the error dynamics as follows:

$$
\begin{gathered}
\dot{e}_{1}=e_{2}+u_{1}(t), \\
\dot{e}_{2}=\left(n_{1}^{2}-\frac{\ddot{l}}{l}\right) e_{1}-\frac{2}{l} l_{e_{2}}+\frac{2 n_{1} l}{l} e_{3}+2 n_{1} e_{4}+A_{2}-A_{1}+u_{2}(t),
\end{gathered}
$$




$$
\begin{gathered}
\dot{e}_{3}=e_{4}+u_{3}(t), \\
\dot{e}_{4}=-\frac{2 n_{1} i}{l} e_{1}-2 n_{1} e_{2}+\left(n_{1}^{2}-\frac{\ddot{l}}{l}\right) e_{3}-\frac{2}{l} \dot{l}_{e_{4}}+B_{2}-B_{1}+u_{4}(t) .
\end{gathered}
$$

This above error system to be controlled is a linear system with control functions. Thus, let us redefine the control functions as follows:

$$
\begin{gathered}
u_{1}(t)=v_{1}(t), \\
u_{2}(t)=-A_{2}+A_{1}+v_{2}(t), \\
u_{3}(t)=v_{3}(t), \\
u_{4}(t)=-B_{2}+B_{1}+v_{4}(t) .
\end{gathered}
$$

The new system can be expressed as:

$$
\begin{gathered}
\dot{e}_{1}=e_{2}+v_{1}(t), \\
\dot{e}_{2}=\left(n_{1}^{2}-\frac{\ddot{l}}{l}\right) e_{1}-\frac{2}{l} \dot{l} e_{2}+\frac{2 n_{1} \dot{l}}{l} e_{3}+2 n_{1} e_{4}+v_{2}(t), \\
\dot{e}_{3}=e_{4}+v_{3}(t), \\
\dot{e}_{4}=-\frac{2 n_{1} i}{l} e_{1}-2 n_{1} e_{2}+\left(n_{1}^{2}-\frac{\ddot{l}}{l}\right) e_{3}-\frac{2}{l} \dot{l} e_{4}+v_{4}(t) .
\end{gathered}
$$

The system (23)-(26) to be controlled is a linear system with a control input $v_{i}(t)(i=1 . .4)$ as function $e_{i}(i=1 . .4)$. As long as these feedbacks stabilize the system $e_{i}(i=1 . .4)$ converge to zero as time t tends to infinity. This shows that the master and the slave system are synchronized with active control techniques. There are many possible choice for the control $v_{i}(t)(i=1 . .4)$. Now Choosing

$$
\left[\begin{array}{l}
v_{1}(t) \\
v_{2}(t) \\
v_{3}(t) \\
v_{4}(t)
\end{array}\right]=A\left[\begin{array}{l}
e_{1} \\
e_{2} \\
e_{3} \\
e_{4}
\end{array}\right], \text { where } A=\left[\begin{array}{cccc}
-1 & -1 & 0 & 0 \\
-\left(n_{1}^{2}-\frac{\ddot{l}}{l}\right)\left(-1+\frac{2 i}{l}\right) & -\frac{2 n_{1} i}{l} & -2 n_{1} \\
0 & 0 & -1 & -1 \\
\frac{2 n_{1} i}{l} & 2 n_{1} & -\left(n_{1}^{2}-\frac{\ddot{l}}{l}\right) & \left(-1+\frac{2 \dot{l}}{l}\right)
\end{array}\right]
$$


is a square constant matrix of order four to be determined. Hence the system (23)-(26) can be define as

$$
\left[\begin{array}{l}
\dot{e_{1}} \\
\dot{e_{2}} \\
\dot{e_{3}} \\
\dot{e_{4}}
\end{array}\right]=B\left[\begin{array}{l}
e_{1} \\
e_{2} \\
e_{3} \\
e_{4}
\end{array}\right], \text { where } B=\left[\begin{array}{cccc}
-1 & 0 & 0 & 0 \\
0 & -1 & 0 & 0 \\
0 & 0 & -1 & 0 \\
0 & 0 & 0 & -1
\end{array}\right]
$$

with eigen values having negative real parts. This implies that $\lim _{t \rightarrow \infty}\left|e_{i}\right|=0 ; i=1,2,3,4$ and according to the Lyapunov stability theory and the Routh-Hurwitz criteria, complete synchronization is achieved between the master and slave systems.

Let us consider an example of Mars-Earth system in the restricted three body problem in which the inner primary is taken as the Earth and outer primary is the Mars and use the astrophysical data the simulation results for uncoupled system are given in figures 3a, 4a, 5a, 6a and controlled system of that are shown in figures 3b, 4b, 5b and 6b respectively.

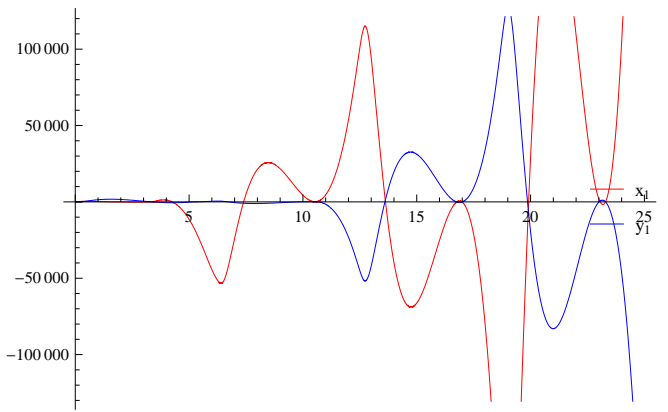

(a) Uncontrolled states.

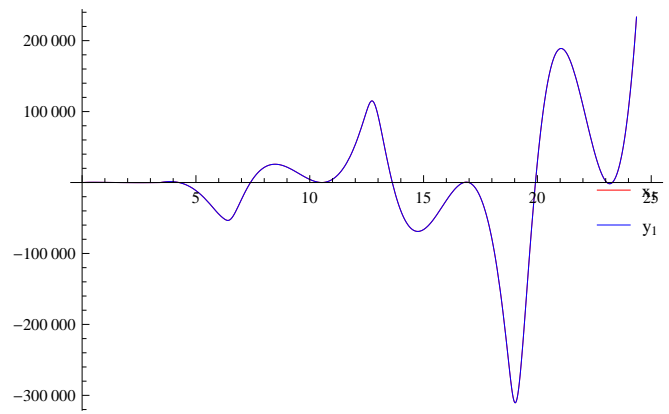

(b) Synchronized states.

Fig. 3: Time series of $x_{1}[t], y_{1}[t]$ (time in second).

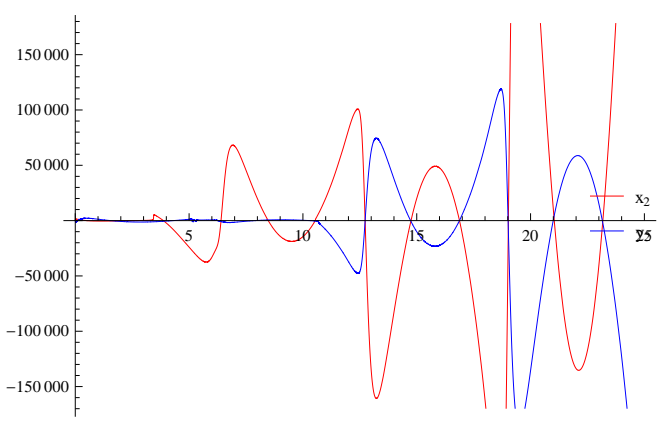

(a) Uncontrolled states.

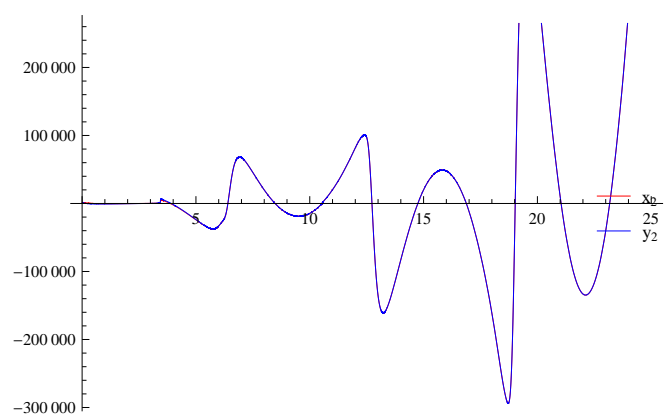

(b) Synchronized states.

Fig. 4: Time series of $x_{2}[t], y_{2}[t]$ (time in second). 


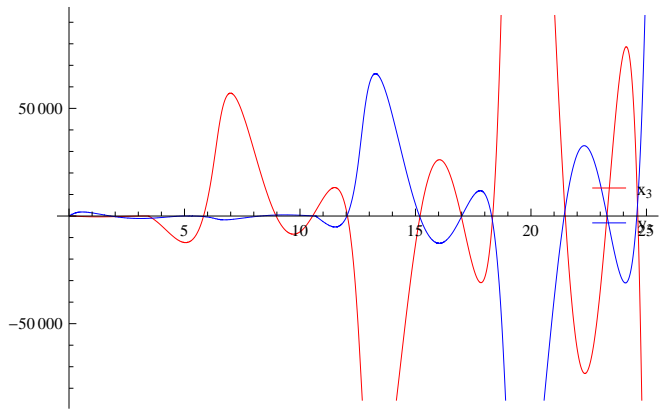

(a) Uncontrolled states.

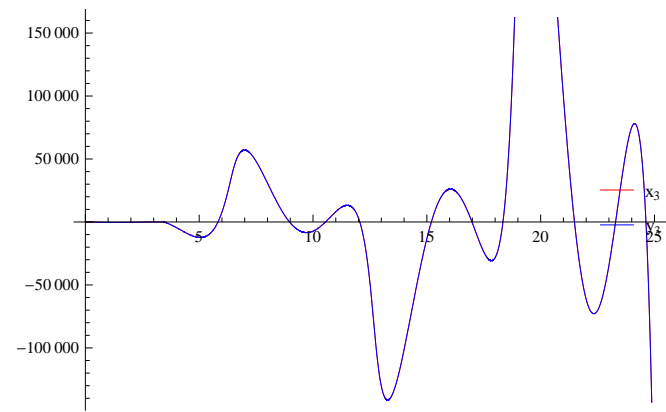

(b) Synchronized states.

Fig. 5: Time series of $x_{3}[t], y_{3}[t]$ (time in second).

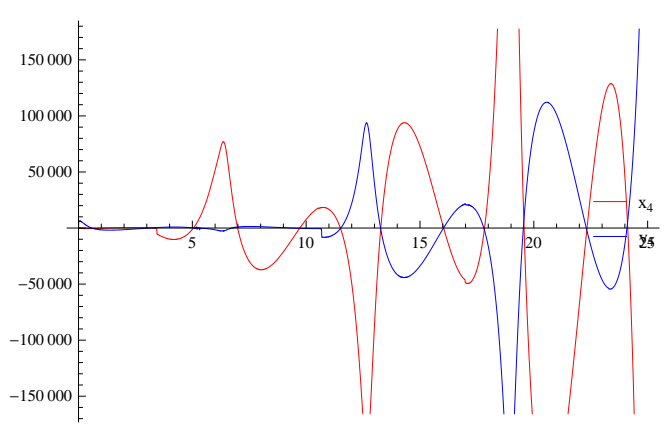

(a) Uncontrolled states.

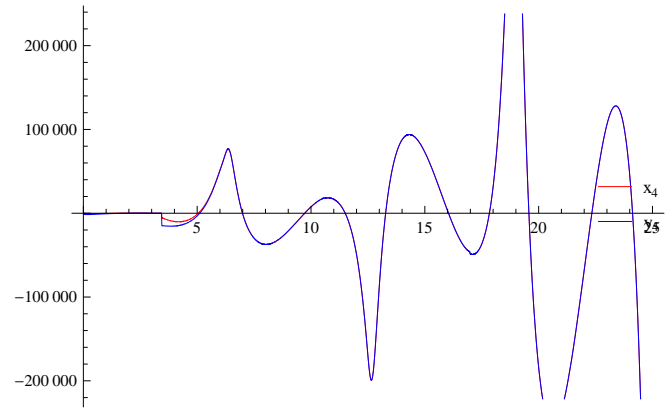

(b) Synchronized states.

Fig. 6: Time series of $x_{4}[t], y_{4}[t]$ (time in second).

\section{Conclusion}

In this article authors formulated the equation of motion when the primaries are moving in orbit- orbit resonance and investigated the complete synchronization behavior of Mars-Earth system, via active control technique based on Lyapunov stability theory and Routh-Hurwitz criteria. Here two systems (master and slave) are completely synchronized evolving from different initial conditions.

\section{Competing interests}

The authors declare that they have no competing interests.

\section{Authors' contributions}

All authors have contributed to all parts of the article. All authors read and approved the final manuscript. 


\section{References}

[1] Ahmad,I., Saaban, A. B., Ibrahim, A. B., Al-Hadhrami, S., Shahzad, M. and Al-Mahrouqi, S. H. A research on adaptive control to stabilize and synchronize a hyperchaotic system with uncertain parameters. An International Journal of Optimization and Control: Theories and Applications, 5(2), 51-62 (2015)

[2] Ahmad,I., Saaban, A. B., Ibrahim A., Shahzad, M. and Alsawalha M. Reduced-order synchronization of time-delay chaotic systems with known and unknown parameters. Optik-Int. J. Lig. Ele. Opt., 127(13), 5506-5514 (2016)

[3] Arif, M. Complete synchronization antisynchronization and hybrid synchronization of restricted three body problem when bigger primary is oblate spheroid and smaller primary is ellipsoid. Int. J. of Adv. in App. Math. and Mech., 4(3), 32-44 (2017)

[4] Bai E.W., Lonngren K.E., Synchronization of two Lorenz systems using active control,. Chaos, Solitons and Fractals, 8, 51-58 (1997)

[5] Birkhoff, G. D. The restricted problem of three bodies. Rend. Circ. Mat. Palermo, 39, (1915)

[6] Bhatnagar, K. B. Periodic orbits of collision in the restricted problem of three bodies in a three-dimensional co-ordinate system. Ind.J.Pure Appl. Math., 3(1), 101-117 (1969)

[7] Bhatnagar, K. B. and Chawla, J. M. A study of the Lagrangian points in the photogravitational restricted three body problem . Ind.J.Pure Appl. Math., 10(11), 1443-1451 (1979)

[8] Codreanu, S. Synchronization of spatiotemporal nonlinear dynamical systems by an active control. Chaos, Solitons and Fractals, 15, 507-510 (2003)

[9] Deprit A. and Price J. F. The computation of characteristic exponents in the planar restricted three body problem. Astro. J., 70, 836-849 (1964)

[10] Euler, L. Un corps etant attire en raison reciproque quarree des distances vers deux points fixes donnes. Mem. Berlin, 228 (1760)

[11] Haeri M., Emadzadeh A., Synchronizing different chaotic systems using active sliding mode control Chaos, Solitons and Fractals, 1, 119-129 (2003)

[12] Hill, G. W. Reasearches in the lunar theory. Am. J. Math. , 1, (1878)

[13] Hadjidemetriou, J. D. Asteroid motion near the 3:1. Celest Mech. And dyn. Astron., 56(1-2), 201-219 (1993)

[14] Khan, A., Shahzad, M. Chaos Synchronization in a Circular Restricted Three Body Problem Under the Effect of Radiation. Chaos and Complex Systems, 59-68 (2012)

[15] Khan, A., Tripathi, P. Synchronization, Anti-Synchronization and Hybrid- Synchronization Of A Double Pendulum Under The Effect Of External Forces. International Journal Of Computational Engineering Research, 3(1) 166-176 (2013)

[16] Khan, A., Rimpi Pal. Complete synchronization, anti-synchronization and hybrid synchronization of two identical parabolic restricted three body problem. Asian Journal of Current Engineering and Maths, 2(2) 118-126 (2013)

[17] Lagrange, J Ocuvres 14, vols Gauthier-Villars, Paris (1772)

[18] Lei, Y., Xu, W. and Xie, W. Synchronization of two chaotic four-dimensional systems using active control. Chaos, Solitons and Fractals, 32, 1823-1829 (2007)

[19] Liu, Z. Several theoretical problems for synchronization. Ziran Za Zhi, 26(5), 298-300 (2004)

[20] Lu L., Zhang C., Guo Z. A., Synchronization between two different chaotic systems with non linear feedback control, Chinese Physics 16-6(2007), 1603-1607.

[21] Mahmoud, G. M., Aly, S. A. and Farghaly, A. A. On chaos synchronization of a complex two coupled dynamos system. Chaos, Solitons and Fractals, 33, 178-187 (2007)

[22] Ott., Grebogi C., Yorke J. A.,Controlling Chaos, Physics Rev. Lett., 64(1990), 1196-1199.

[23] Park J. H., Chaos synchronization between two different chaotic dynamical systems. Chaos, Solitons and Fractals, 27-2, 549-554 (2006)

[24] Pecora L. M., Carroll T. L.,Synchronization in chaotic systems, Physics Rev. Lett., 64(1990), 821-824.

[25] Poincare, H. Sciences et Methode, (1908)

[26] Sahoo, S. k. and Ishwar, B. Stability of the collinear equilibrium points in the generalized photogravitational elliptical restricted three body problem. Bull Astr. Soc. India , 28, 579-586 (2000) 
[27] Sharma, R. K. and Subbarao collinear equilibria and their characteristic exponents in the restricted three body problem when the primaries are oblate spheroids. Celestial Mechanics, 12, 189-201 (1975)

[28] Sharma, R. K., Taqvi, Z. A. and Bhatnagar, K.B. Existence and stability of the libration point of the restricted three body problem when the bigger primary is a triaxial body and a source of radiation. Indian J pure appl. Math., 32(2), 255-266 (2001)

[29] Szebehely, V. Theory of Orbits., Academic press, New York, (1967)

[30] Ucar, A., Bai, E. W. and Lonngren, K. E. Synchronization of chaotic behavior in nonlinear Bloch equations. Phys. Lett. A , 314, 96-101 (2003)

[31] Ucar, A., Bai, E. W. and Lonngren, K. E. Chaos synchronization in RCL-shunted Josephson junction via active control. Chaos, Solitons and Fractals, 31, 105-111 (2007)

[32] Vincent, U. E. Synchronization of Rikitake chaotic attractor via active control. Phys. Lett. A , 343, 133-138 (2005)

[33] Wang Y., Guan Z. H.,Wang H. O., Feedback and adaptive control for the synchronization of Chen system via a single variable Phys. Lett. , 312-1, 30-40 (2003)

[34] Winter, A. The analytical foundation of Celestial Mechanics, Princeton (1941)

[35] Whittaker, E.T. A treatise on the analytical dynamics of particles and rigid bodies, Cambridge Univ., London and New York, original first ed., (1904) 